\title{
Resuming elective surgery in the time of COVID-19: a safe and comprehensive strategy
}

\author{
Khaled Al-Omar ${ }^{1}$ - Sohail Bakkar ${ }^{1}$ (1) $\cdot$ Laith Khasawneh ${ }^{1} \cdot$ Gianluca Donatini $^{2} \cdot$ Paolo Miccoli $^{3}$
}

Received: 18 May 2020 / Accepted: 22 May 2020 / Published online: 3 June 2020

(C) Italian Society of Surgery (SIC) 2020

\begin{abstract}
The 2019 novel corona virus and the disease it causes (COVID-19) is a public health crisis that has profoundly modified the way medical and surgical care is delivered. Countries around the globe had a variable initial response to the COVID-19 pandemic from imposing massive lock downs and quarantine to surrendering to herd immunity. However, healthcare bodies worldwide recognized early on that a triumph against COVID-19 could only be achieved by maintaining the infrastructure of healthcare systems and their capacity to accommodate a potentially overwhelming increase in critical patient care needs. Therefore, they reacted by restricting medical care to emergency cases and postponing elective surgical procedures in all disciplines. The priority was made for treatment of COVID-19 patients and emergency cases. Nevertheless, the battle against the COVID-19 pandemic is still ongoing. In the absence of vaccines or effective drug treatments, its timeline remains uncertain and it cannot be forecast how long healthcare systems will need to cope with it in managing inpatient and outpatient services. Accordingly, extreme measures and restriction may become a recipe for a disaster in the context of the potential adverse health implications imposed by delaying timely medical and surgical care. Therefore, restrictive measures should be substituted with a comprehensive surgical and medical care strategy. One that provides a safe balance between the prevention of COVID-19 and the delivery of essential surgical care. This article provides an overview on how to safely deliver essential surgical care in the time of COIVD-19.
\end{abstract}

Keywords COVID-19 $\cdot$ SARS-CoV-2 $\cdot$ Pandemic $\cdot$ Elective surgery

\section{Introduction}

On the 31st of December 2019, the first case of a novel corona virus disease was reported in Wuhan, China, and the virus was named severe acute respiratory syndrome coronavirus 2 (SARS-CoV-2) [1]. The 2019 novel corona virus and the disease it causes was named COVID-19 by the world health organization (WHO) in February 2020, and in March it declared COVID-19 a global pandemic [2]. To date, more than 4 million cases have been reported with over 300,000 deaths [3]. According to the Chinese center for disease

Sohail Bakkar

sohail.bakkar@gmail.com

1 Department of Surgery, Faculty of Medicine, The Hashemite University, Zarqa 13133, Jordan

2 Department of General and Endocrine Surgery, University of Poitiers, Poitiers, France

3 Department of Surgical, Medical, Pathology, and Critical Care, the University of Pisa, 56124 Pisa, Italy control and prevention, COVID-19 despite being highly contagious, tends to be asymptomatic or mildly symptomatic in most instances [4]. Nevertheless, acute respiratory distress syndrome (ARDS), and death remain possible outcomes. In this period of uncertainty, it becomes essential to maintain a healthcare system's capacity to support a potentially overwhelming increase in critical patient care needs. The American College of Surgeons (ACS) called to prioritize appropriate resource allocation as it relates to elective invasive procedures and minimize the use of essential items required to care for patients [5]. It also emphasized the importance of doing that since many patients tend to be asymptomatic, and serve as an invisible threat to other patients, and healthcare providers.

Globally, healthcare authorities have reacted by restricting medical care to emergency cases and postponing elective surgical procedures of all types. The priority has been made for the treatment of COVID-19 patients and emergency cases, in anticipation of a possible need for hospital beds and resources [6].The unpredictability of the timeline 
of COVID-19 suggests that patients may be deprived of access to needed surgical care, likely for many months. The potential outcome of delay in necessary elective surgical procedures may have a more detrimental impact on patients' health compared to that of COVID-19 itself.

The purpose of this article is to highlight how extreme measures and restrictions could be transformed into a comprehensive and safe surgical strategy. One that provides a safe balance between the prevention of COVID-19 transmission and the delivery of surgical care.

\section{A proposed safe and comprehensive surgical strategy}

Overwhelming the healthcare sector with critically ill patients will undeniably have a directly proportional impact on mortality. Accordingly, flattening the pandemic curve has been the goal of health care professionals worldwide [7]. A potentially growing number of patients that might need to visit emergency departments and require admission, along with potential shortages in healthcare workers due to viral exposures justify the imposed mitigations and restrictions. Nevertheless, historically, pandemics have had an unpredictable timeline and could have more than one spike in cases over their course [8]. The influenza pandemic of 1918, aka the Spanish flu, occurred in three waveforms and caused about 50 million deaths between the years 1918 and 1919 . The second and third waves were more severe than the first, and over 500 million people were infected [9]. Likewise, the 1889 pandemic also occurred in three waveforms over a 3 -year period [10]. As for COVID-19, in the absence of vaccines or effective drug treatments, and the possibility of several wave forms its timeline remains uncertain and it cannot be forecast how long healthcare systems will need to cope with it in managing inpatient and outpatient services. This mandates strategic planning to keep essential necessities available in the safest possible way. We, therefore, propose a four-tier comprehensive surgical care scheme designed to fit COVID-19 and any potential outbreak in the future. The proposed strategy allows for the safe performance of elective surgical procedures while minimizing the risk of viral transmission. The four categories of the proposed surgical strategy include (1) The isolation of COVID-19 treatment facilities (2) The prioritization of surgical cases (3) Screening surgical patients for COVID-19, and (4) Maintaining a safe and clean hospital environment.

\section{Isolating COVID-19 treatment facilities}

Countries around the world have adopted different approaches in fending-off the COVID-19 outbreak. In-hospital isolation of all infected patients regardless of disease severity was adopted by countries with low numbers of cases [11]. Specific regional hospitals were isolated for the sole purpose of hosting COVID19 patients. This strategy could prove to be difficult in regions, where numbers could exceed the capacity of hospitals. Therefore, isolating specific facilities for the treatment of COVID-19 patients will keep other facilities untainted, thereby decreasing the risk of viral transmission. Isolation should also include healthcare providers and personnel. In regions, where the numbers of patients requiring hospital care exceed capabilities and resources, careful consideration of postponing elective surgeries should be undertaken. The dependence of decisions related to reducing non-urgent procedures on each facility's geographic location has also been recognized by both the American Society of Anesthesiologists (ASA) and the American Patient Safety Foundation (APSF) [12].

Estimating the potential effects of a pandemic on a regional resource could be achieved using specialized programs such as the Flu 2.0 and Flu surge 2.0, introduced by Dr. Martin Meltzer from the center of disease control and prevention (CDC) [13, 14]. These programs estimate the potential effects of a pandemic on a region based on its population and available resources [15]. Anticipating the potential sequalae of a pandemic on a specified region could effectively help in proper resource planning and handling including personnel, personal protection equipment (PPE), and intensive care resources.

\section{Prioritizing surgical cases}

The urgency of surgical procedures exists along a continuum. Not all procedures are considered time critical. Therefore, surgeries could be stratified according to indication and degree of urgency bearing in mind that many elective surgical procedures could upgrade in terms of priority at any time and become urgent or emergent. The unpredictable timeline of COVID-19 necessitates developing safe strategies that help guide timely surgical decision making. Elective procedures, from a practical point of view, could be classified into two broad categories: essential and non-essential or discretionary. The former implies that delaying surgical management for an undetermined period is potentially associated with an increased risk of adverse outcomes. While the latter alludes to purely elective procedures that are not timesensitive from a surgical and/or medical perspective. Table 1 provides examples of surgical case-types stratified based on indication and urgency [16].

Resuming elective procedures should be planned in consideration with the capabilities of healthcare facilities. Giving priority to more urgent procedures and expanding to include less urgent procedures in the context of resource availability. 
Table 1 Surgical case-types stratified based on indication and urgency

\begin{tabular}{lll}
\hline Indication & Time frame for action & Example \\
\hline Emergent & $<1 \mathrm{~h}$ & Life-threatening emergencies \\
& & Acute exsanguination \\
& & Acute vascular injury or occlusion \\
& & Aortic dissection \\
& & Emergency caesarian section \\
Urgent & $<24 \mathrm{~h}$ & Compartment syndrome \\
& & Necrotizing fasciitis \\
& & Acute appendicitis \\
& Septic arthritis \\
Urgent elective & Open fractures \\
& Bleeding pelvic fractures \\
& & Femur shaft fractures \\
Essential elective & Cardiothoracic procedures \\
& & Closed fractures \\
& & Scheduled caesarian section \\
& & Wound closure/skin grafts or flaps \\
& & Cancer surgery or biopsies \\
& & Hernia repair \\
& & Hysterectomy \\
& & Cosmetic surgery \\
& & Sports surgery \\
& & Infertility procedures \\
\hline
\end{tabular}

Modified from the work of Stahel PF [16]

\section{Screening surgical patients for COVID-19}

ARDS, myocarditis, and renal injury are some of the potentially expected postoperative adverse consequences in COVID-19 patients [17]. Therefore, well designed screening protocols for surgical patients are essential to ensure the safety of not only healthcare providers, but also patients themselves. The ASA and the APSF have published a joint statement on non-urgent care during the COVID-19 outbreak [12]. Their recommendations were complementary to the CDC COVID-19 healthcare facility guidance [18]. The joint statement advised identifying the prevalence of COVID-19 in populations prior to the implementation of any perioperative testing protocol.

We propose a modified screening protocol listed under two broad headings based on the regional prevalence of COVID-19. Prevalence is classified as either high or low based on calculations comparing the prevalence of influenza-like illnesses to that of previous seasons of flu [19].

I. Local or regional presence (high priority screening)

All patients should be screened for symptoms prior to presenting to the hospital. a. Symptomatic patients should have their surgery postponed and should undergo nucleic acid amplification testing at a COVID-19 dedicated facility.

b. Asymptomatic patients should undergo nucleic acid amplification testing at the surgical center and if positive, surgery should be postponed until they become non-infectious.

Patients who have negative test results can undergo surgery after clearance from health care provider.

II. Low or no regional prevalence (low priority testing)

All patients should be screened for symptoms before presenting to the hospital. Patients reporting symptoms should be referred for nucleic acid amplification testing:

a. Test positive patients should have their surgery postponed until they become non-infectious.

b. Test negative patients can undergo surgery if cleared by healthcare providers per medical status.

Asymptomatic patients in areas of low or no prevalence should be able to undergo surgery with no further testing.

The CDC COVID-19 healthcare facility guidance recommends discontinuing transmission-based precautions in symptomatic patients based on either a test-based or 
a symptom-based strategy. While adopting a test-based or a time-based strategy for asymptomatic patients [18].

\section{Maintaining a safe and clean hospital environment}

Due to the likelihood of false positive and false negative testing results, it is necessary to assume that everyone is infected. Implementing maximum safety precautions will reduce the likelihood of viral transmission [20]. Preventing nosocomial spread is a priority for all health care facilities. Therefore, healthcare providers including doctors and nurses should always use PPE. Operating room staff should wear surgical face masks and eye coverings (droplet precautions) for standard operative cases. Aerosolized procedures should be performed using maximum PPE's (N95 masks, eye protection, gloves, and gowns) [20]. Rationalized consumption of PPE should also be considered in anticipation of any overwhelming need in the future. Cleaning and disinfection protocols should be updated and reviewed continuously, especially when disinfecting after infected patients [21].

Visitors should be kept to a minimum and alternative communication platform could be set up to help patients keep in touch with loved ones during their stay. Visitors, if allowed, should also be screened for any flu like symptoms before being granted visiting permission. Furthermore, they should be restricted to certain areas and should wear masks, gloves, and gowns.

In-hospital chaperones accompanying patients should be screened like any admitted patient, by clinical history and nucleic acid amplification testing. Positive chaperones should be prevented from accompanying patients until they are non-infectious according to CDC transmission guidelines [18].

Keeping a safe and clean hospital environment is necessary for resuming elective surgical procedures. Viral transmission is a major concern when considering nonurgent interventions during a pandemic. However, in some instances the consequences resulting from a delay in delivering surgical care may outweigh those of the viral infection itself.

\section{Conclusion}

Our proposed strategy is designed to help guarantee the timely delivery of surgical care in a comprehensive, safe, and well-balanced manner during the COVID-19 outbreak. This strategy may be subject to future modifications and adjustments that correspond to new developments in the COVID-19 outbreak.
Author contributions All authors contributed to the study concept and design, data interpretation, drafting, final approval, and accountability for all aspects of the work

Funding This study was not funded by any grant.

\section{Compliance with ethical standards}

Conflict of interest The authors declare that they have no conflict of interest.

Ethical approval Ethical committee approval was waived because of the type of this article.

Informed consent Informed consent is not applicable to this type of articles.

Data availability statement Not applicable because of the type of this article.

\section{References}

1. Ghebreyesus TA (2020) WHO Director-General's opening remarks at the media briefing on COVID-19-2020. https://www. who.int/dg/speeches/detail/who-director-general-s-opening-remar ks-at-the-media-briefing-on-covid-19. Accessed 11 Mar 2020

2. "WHO Director-General's opening remarks at the media briefing on COVID-19- 2020" (2020) https://www.who.int/dg/speeches/ detail/who-director-general-s-opening-remarks-at-the-media-brief ing-on-covid-19. Accessed 11 Mar 2020

3. COVID-19 Corona virus pandemic (2020) worlds meters info. https://www.worldometers.info/coronavirus. Accessed 15 May 2020

4. Wu Z, McGoogan JM (2020) Characteristics of and important lessons from the coronavirus disease 2019 (COVID-19) outbreak in China: summary of a report of 72314 cases from the Chinese Center for Disease Control and Prevention. JAMA 323:12391242. https://doi.org/10.1001/jama.2020.2648

5. American College of Surgeons (ACS) (2020) COVID-19 update: guidance for triage of non-emergent surgical procedures. https ://www.facs.org/covid-19/clinical-guidance/triage. Accessed 17 March 2020

6. Commins J (2020) Surgeon general urges providers to consider stopping all elective surgeries-hospitals push back. Health Leaders. 2020. https://www.healthleadersmedia.com/clinical-care/ surgeon-general-urges-providers-consider-stopping-elective-surge ries-hospitals-push. Accessed 17 Mar 2020

7. Matrajt L, Leung T (2020) Evaluating the effectiveness of social distancing interventions to delay or flatten the epidemic curve of coronavirus disease. Emerg Infect Dis. https://doi.org/10.3201/ eid2608.201093

8. Taubenberger JK, Morens DM (2006) 1918 Influenza: the mother of all pandemics. Emerg Infect Dis. https://doi.org/10.3201/eid12 01.050979

9. Frost WH (1920) Statistics of influenza morbidity. Public Health Rep 35:584-597. https://doi.org/10.2307/4575511

10. Jordan EO (1927) Epidemic influenza; A survey. Chicago: American Medical Association. https://www.oalib.com/references /26221. Accessed 17 Mar 2020

11. Younes A (2020) How Jordan is flattening its COVID-19 curve. Encyclopedia News. https://encyclopedic.co.uk/2020/04/22/howjordan-is-flattening-its-covid-19-curve. Accessed 22 Apr 2020 
12. American Society of Anesthesiologists (ASA) and the Anesthesia Patient Safety foundation (APSF) (2020) ASA and APSF Joint Statement on Perioperative Testing for the COVID-19 Virus. https ://www.asahq.org/about-asa/newsroom/news-releases/2020/04/ asa-and-apsf-joint-statement-on-perioperative-testing-for-thecovid-19-virus. Accessed 29 Apr 2020

13. Meltzer MI, Cox NJ, Fukuda K (2007) Centers for disease control. National vaccine program office FluAid. http://www.cdc.gov/flu/ tools/fluaid/. Accessed 11 Oct 2007

14. Meltzer MI, Cox NJ, Fukuda K (2007) Centers for disease control and prevention. FluSurge software. http://www.cdc.gov/flu/tools/ flusurge/. Accessed 11 Oct 2007

15. Mareiniss DP, Hirshon JM, Thibodeau BC (2009) Disaster planning: potential effects of an influenza pandemic on community healthcare resources. Am J Disaster Med 4:163-171

16. Stahel PF (2020) How to risk-stratify elective surgery during the COVID-19 pandemic? Patient Saf Surg 14:8. https://doi. org/10.1186/s13037-020-00235-9

17. Aminian A, Safari S, Razeghian-Jahromi A, Ghorbani M, Delaney CP (2020) COVID-19 outbreak and surgical practice: unexpected fatality in perioperative period. Ann Surg. https://doi.org/10.1097/ SLA.0000000000003925 (ahead of print)

18. Centers for Disease Control and Prevention, National Center for Immunization and Respiratory Diseases (NCIRD), Division of Viral Diseases (2020) Discontinuation of transmission-based precautions and disposition of patients with COVID-19 in healthcare settings (Interim Guidance). https://www.cdc.gov/coronaviru s/2019-ncov/hcp/disposition-hospitalized-patients.htm. Accessed 28 Apr 2020

19. Centers for Disease Control and Prevention, National Center for Immunization and Respiratory Diseases (NCIRD) (2016) Overview of influenza surveillance in the United States. https://www. cdc.gov/flu/pdf/weekly/overview-update.pdf. Accessed 10 May 2020

20. Centers for Disease Control and Prevention, National Center for Immunization and Respiratory Diseases (NCIRD) (2020) Transmission-based precautions. https://www.cdc.gov/infectioncontro 1/basics/transmission-based-precautions.html. Accessed 10 May 2020

21. Centers for Disease Control and Prevention, National Center for Immunization and Respiratory Diseases (NCIRD) (2020) Cleaning and disinfecting your facility. https://www.cdc.gov/coron avirus/2019-ncov/community/disinfecting-building-facility.html. Accessed 10 May 2020

Publisher's Note Springer Nature remains neutral with regard to jurisdictional claims in published maps and institutional affiliations. 